\title{
Desmoplastic melanocytic nevi with lymphocytic aggregates
}

Desmoplastic melanocytic nevi can be difficult to distinguish from desmoplastic melanoma. The presence of lymphocytic aggregates in association with a sclerosing melanocytic proliferation is commonly regarded as a feature in support of a diagnosis of desmoplastic melanoma. However, the finding is not specific for melanoma. Herein we report six cases of sclerosing melanocytic nevi with associated lymphocytic aggregates. They occurred in five women and one man, ranging in age from 11 to 61 years. Three lesions were sclerosing Spitz nevi; one was an amelanotic sclerosing blue nevus, one an acquired intradermal sclerosing nevus, and one was a congenital compound melanocytic nevus with sclerosis of its dermal component. The lesions were interpreted as benign, i.e. melanocytic nevi, because of their histopathologic attributes (symmetric silhouette, benign cytologic features) and results from immunohistochemical studies (all lesions strongly expressed Melan-A and p16) and fluorescence in situ hybridization (FISH). Three lesions tested by FISH lacked copy number changes of $11 p, 6 q$ or $6 p$. None of the lesions recurred. The cases highlight that contextual information is essential for the diagnosis of desmoplastic melanoma and sclerosing nevus. The presence of lymphocytic aggregates per se does not prove that a sclerosing melanocytic proliferation is malignant.

Keywords: dermatopathology, Histology, Melan-A, melanocytic lesions

Kiuru M, Patel RM, Busam KJ. Desmoplastic melanocytic nevi with lymphocytic aggregates.

J Cutan Pathol 2012; 39: 940-944. (C) 2012 John Wiley \& Sons A/S.

\section{Maija Kiuru', Rajiv M. Patel ${ }^{2}$ and Klaus J. Busam ${ }^{3}$}

${ }^{1}$ Department of Dermatology, Weill Medical College of Cornell University, New York, NY, USA,

2Department of Pathology and Dermatology, University of Michigan Medical Center, Ann Arbor, MI, USA, and

${ }^{3}$ Department of Pathology, Memorial Sloan-Kettering Cancer Center, New York, NY, USA

Klaus J. Busam, MD

Department of Pathology, Memorial

Sloan-Kettering Cancer Center, 1275 York Ave,

New York, NY 10065, USA

Tel: +12126395679

Fax: +12127173203

e-mail: busamk@mskcc.org

Accepted for publication June 26, 2012
Desmoplastic melanoma is a rare variant of cutaneous melanoma characterized by a prominent collagenous stroma. ${ }^{1,2}$ It tends to affect chronically sun-damaged skin of elderly Caucasians, but can also develop in skin of younger individuals without significant solar elastosis. ${ }^{1-7}$ Desmoplastic melanoma is clinically characterized by a higher tendency for local recurrence and less frequent regional lymph node involvement than conventional melanoma. ${ }^{5-7}$

Desmoplastic melanoma can be difficult to diagnose clinically and histopathologically. 1,2,8,9 Failure to recognize desmoplastic melanoma amounts to a significant proportion of medical malpractice claims related to melanoma. ${ }^{10}$ Under the microscope, desmoplastic melanoma can be confused with non-melanocytic spindle cell tumors, such as spindle cell squamous cell carcinoma or a sarcoma. ${ }^{1,8,9}$ It may simulate the appearance of a scar. A particularly diagnostic challenge is its distinction from a sclerosing melanocytic nevus, especially on a partial shave biopsy of a clinically indistinct lesion. , $^{1,11-15}$

A number of pathologic parameters are useful for the recognition of desmoplastic melanoma and its distinction from a sclerosing melanocytic nevus. ${ }^{1,11-15}$ One feature that is commonly mentioned as helpful for the diagnosis of desmoplastic melanoma is the presence of lymphocytic aggregates. However, such lymphocytic aggregates are not 


\section{Desmoplastic melanocytic nevi with lymphocytic aggregates}

unique for melanoma. They can be seen in other fibrosing lesions, including sclerosing melanocytic nevi. Herein we report six cases of sclerosing melanocytic nevi with lymphocytic aggregates to document the occurrence of this association. Our findings confirm limits in the specificity of the diagnostic value of lymphocytic aggregates.

\section{Materials and methods}

Case selection

The cases were collected from the clinical material of two authors with an interest in this topic. No diagnostically ambiguous cases were included.

\section{Immunohistochemical studies}

Five micron thick sections from formalin-fixed and paraffin-embedded tissue blocks were taken and analyzed immunohistochemically using standard avidin-biotin procedure. An automated IHC system (Ventana BenchMark XT, Ventana Medical Systems, Inc., Tucson, AZ, USA) using an alkaline phosphatase method and a red chromogen was used. The antibodies tested included S100 protein (Cell Marque, Hot Springs, AR, USA), A103 (Dako, Glostrup, Denmark), p16 (Dako), CD3 (Dako), CD79a (Dako), CD4 (Ventana Medical Systems, Inc.) and CD8 (Ventana Medical Systems, Inc.). They were used according to the manufacturer's instructions.

\section{Fluorescence in situ hybridization}

Fluorescence in situ hybridization (FISH) testing was performed using four probes targeting Ras Responsive Element-Binding Protein-1 (Vysis ${ }^{\circledR}$ LSI ${ }^{\circledR}$ RREB1-Spectrum Red), myeloblastosis $\left(V_{y s i s}{ }^{\circledR}\right.$ LSI ${ }^{\circledR}$ MYB-S Gold), cyclin D1 or chromosome 11q (Vysis ${ }^{\circledR}$ LSI ${ }^{\circledR}$ CGND1-Spectrum Green $\left.^{\mathrm{TM}}\right)$, and centromeric enumeration probe control for chromosome $6\left(\mathrm{Vysis}^{\circledR} \mathrm{LSI}{ }^{\circledR}\right.$ GEP6Spectrum Aqua) from Abbott Molecular, Inc. (Des Plaines, IL, USA). The assay was performed as previously described. ${ }^{16,17}$

\section{Results}

\section{Clinical findings}

The six lesions reported herein affected five female and one male patient. Their ages ranged from 11 to 61 years $($ mean $=31$ years, median $=33$ years $)$. The sites affected included the ear (two lesions), eyelid, chest, back and arm. The biopsies were submitted as rule out nevus vs. fibroma. There has been no recurrence within the available follow-up period (range 3 months to 10 years, mean $=2.5$ years, median $=1.7$ years).

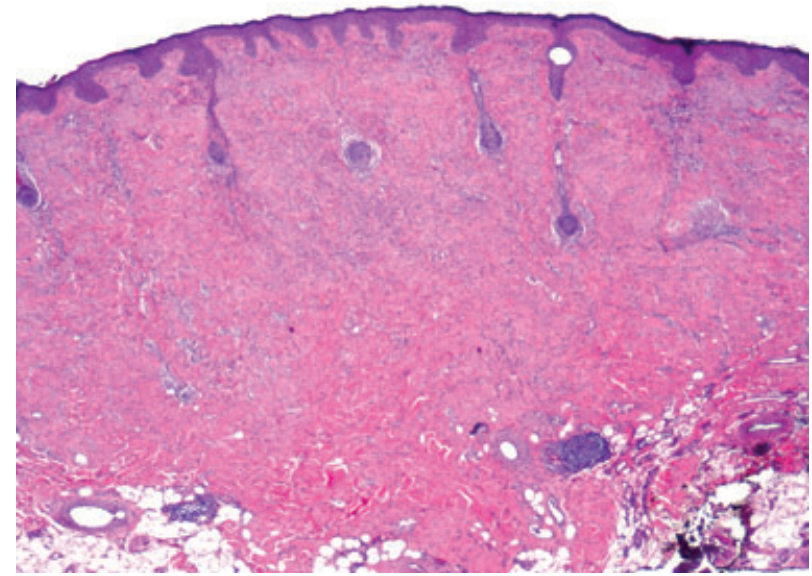

Fig. 1. Wedge-shaped symmetric silhouette of an intradermal paucicellular sclerosing nevus with lymphocytic aggregates in the reticular dermis.

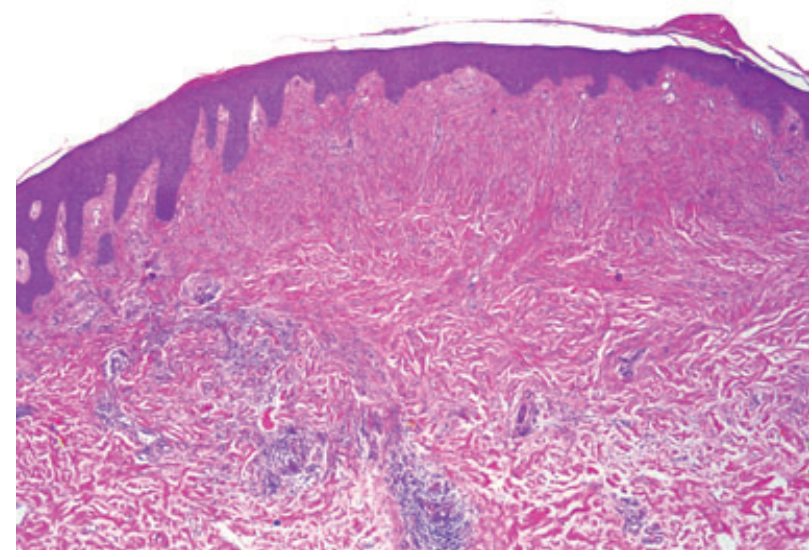

Fig. 2. Compound melanocytic nevus with sclerosis. Nests of melanocytes are present at the dermoepidermal junction and in the superficial dermis. Solitary units of melanocytes predominate within sclerotic dermis. Lymphocytic aggregates are present at the base.

\section{Histopatholgic features}

Three lesions were compound melanocytic nevi. Three were intradermal. Their silhouettes were wedge-shaped and symmetric (Fig. 1). Due to the associated sclerosis they had a 'pink' appearance at scanning magnification. In the cases of the compound nevi, the junctional component showed a predominant nested growth pattern and was peripherally confined to the area overlying the dermal melanocytes (Fig. 2). All lesions showed evidence of maturation with dispersion of cells into solitary units at the base of the dermal nevus component. None of them contained an intradermal melanocyte in mitosis.

Three of the sclerosing nevi show spitzoid cytology (Fig. 3). The remaining three lesions included 


\section{Kiuru et al.}

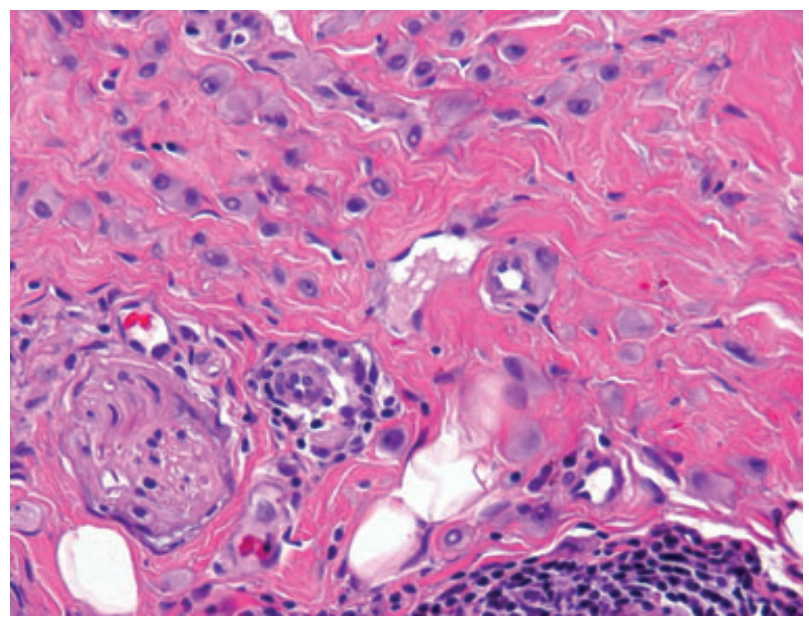

Fig. 3. Sclerosing Spitz nevus with epithelioid melanocytes adjacent to a lymphocytic aggregate.

a sclerosing pauci-melanotic blue nevus, irritated intradermal sclerosing melanocytic nevus and one congenital melanocytic nevus with sclerosis of its dermal component.

The lymphocytic aggregates were typically present in the mid or deep reticular dermis (Figs. 1-3). The number of lymphocytic aggregates per lesion ranged from 1 to 7 (mean $=3$, median $=3$ ). They were composed of small lymphocytes and rare plasma cells.
Immunohistochemical findings

The intradermal spindle cells of all lesions were strongly and diffusely immunoreactive for S100 protein, Melan-A and p16. A typical immunohistochemical profile is illustrated in Fig. 4. In all lesions, the Mib-1 labeling index of intradermal melanocytes was $<2 \%$.

In three cases there was sufficient material to immunophenotype the lymphocytic aggregates. All aggregates were composed of a mixture of $\mathrm{T}$ and B-cells (Fig. 5). The population of T-cells included both CD4 and CD8-positive cells.

\section{Cytogenetic findings}

Three of the six cases were examined cytogenetically by FISH analysis, using four probes to loci on $6 \mathrm{p}$, 6q, 6 cent and 11q and previously published criteria. None of the cases show chromosomal copy number gains or losses diagnostic for melanoma.

\section{Discussion}

Desmoplastic melanoma is a distinct variant of melanoma characterized by prominent stromal fibrosis. ${ }^{1,2}$ Stromal desmoplasia, however, may also be associated with a melanocytic nevus, including conventional ('common acquired'), Spitz or blue
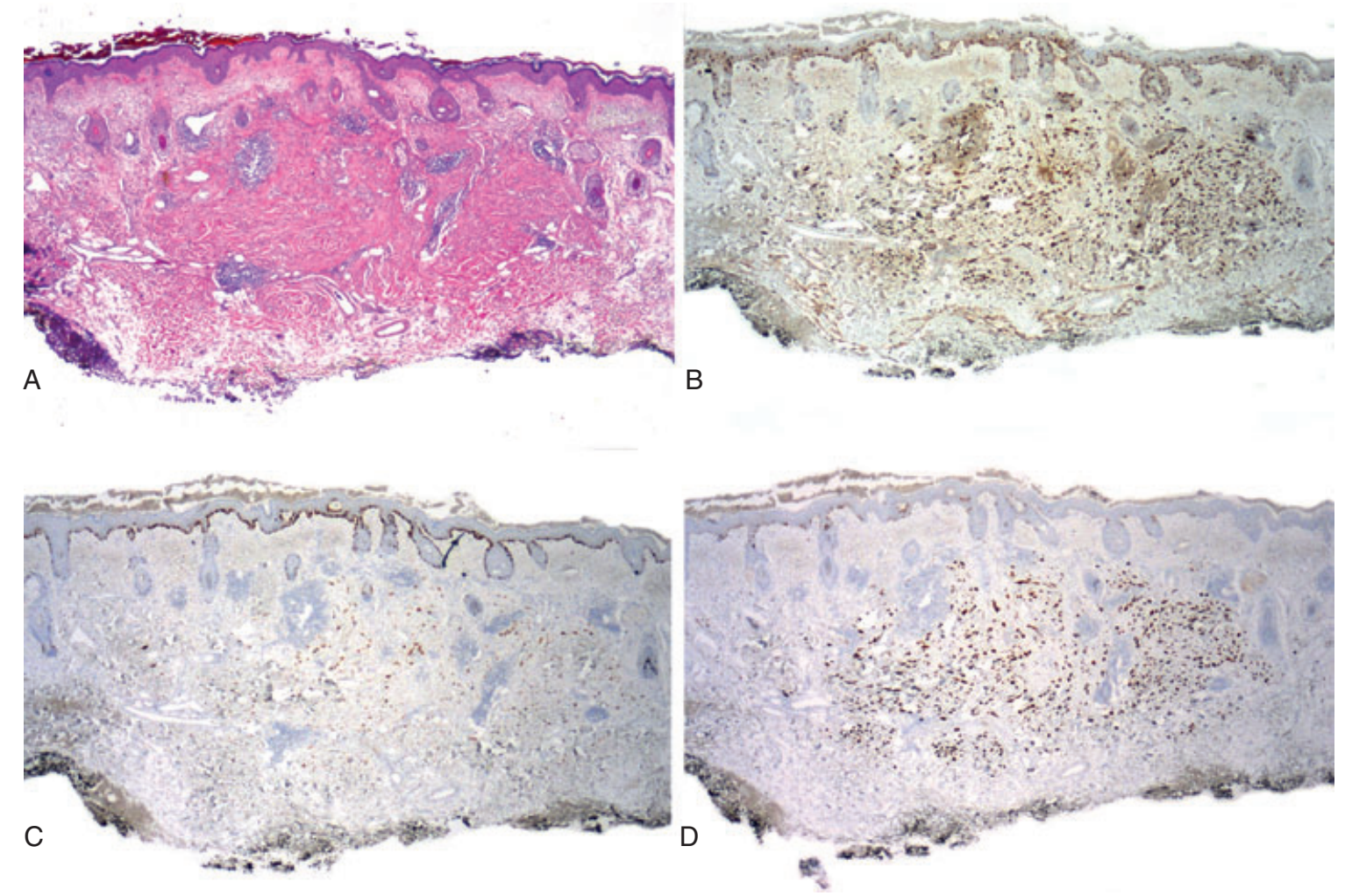

Fig. 4. Sclerosing Spitz nevus with lymphocytic aggregates. A) Hematoxylin and eosin-stained section. B-D) Immunohistochemical staining for $\mathrm{S} 100$ protein $(\mathrm{B})$, melan-A $(\mathrm{C})$ and p16 (D). 


\section{Desmoplastic melanocytic nevi with lymphocytic aggregates}

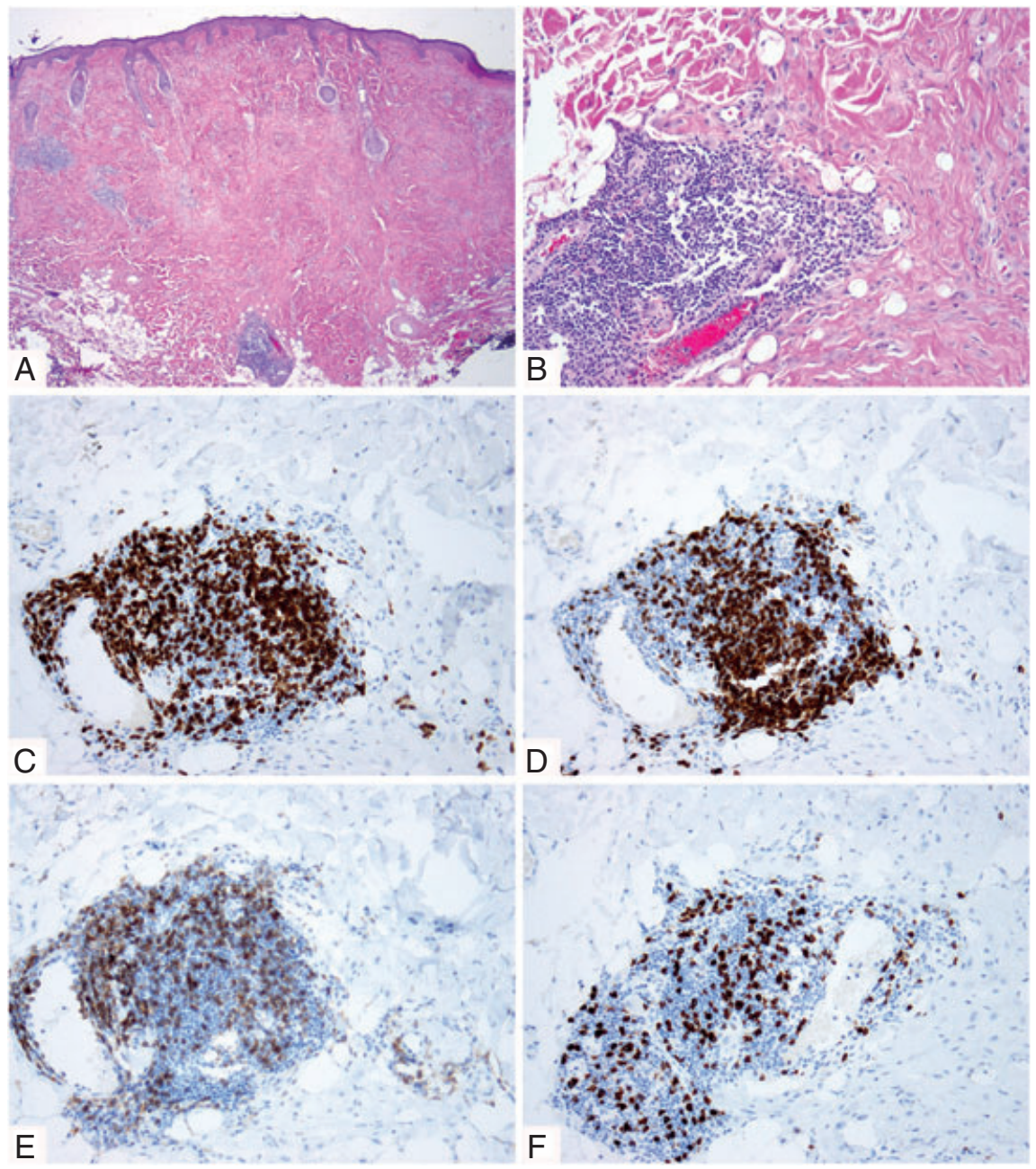

Fig. 5. Immunophenotype of the lymphocytic aggregates. A and B) Sclerosing melanocytic nevus with lymphocytic aggregates (H\&E). Immunohistochemical staining for CD3 (C), GD79a (D), CD4 (E) and GD8 (F).

nevus. ${ }^{11-15}$ Distinguishing desmoplastic melanoma from a desmoplastic or sclerosing melanocytic nevus can be challenging, especially on a small partial biopsy. ${ }^{1,9,11-15}$ Clinical features can be helpful, but are of limited value because of significant overlap.

Histopathologically, the distinction between desmoplastic melanoma and a sclerosing melanocytic nevus usually can be made with confidence, if one is given an adequate excisional biopsy of the lesion that permits evaluation of parameters relevant for the diagnosis. ${ }^{1,11-15}$ If the desmoplastic dermal melanocytic proliferation is accompanied by intraepidermal (in situ) melanoma, the diagnosis is straightforward. However, in approximately one third of desmoplastic melanomas, there is no in situ melanoma detectable within the epidermis. ${ }^{1-3}$ In these cases, one needs to rely entirely on the features of the intradermal melanocytes. Desmoplastic melanoma typically displays an infiltrative asymmetric silhouette and often extends into the subcutis. Sclerosing nevi tend to be symmetric, more broad than deep and confined to the dermis. Desmoplastic melanoma is usually amelanotic. Sclerosing nevi are more likely to contain pigment, at least focally. Desmoplastic melanoma characteristically contains hyperchromatic elongated fusiform melanocytes. Sclerosing nevi, especially sclerosing Spitz's nevi, are more likely to contain epithelioid melanocytes with open chromatin and distinct nucleoli.

Ancillary studies have been forwarded for use in cases in which the distinction of desmoplastic melanoma from a melanocytic nevus is difficult. Commonly used immunohistochemical markers in this setting include Melan-A/Mart- $1,{ }^{18} \mathrm{Ki}-67,{ }^{18}$ and p16. ${ }^{19}$ Desmoplastic melanomas tend to have a higher Ki-67 labeling index than melanocytic nevi and are often negative for Melan-A/Mart-1 and p16, while sclerosing nevi usually lack staining for Ki-67 and tend to be strongly immunoreactive for MelanA/Mart-1 and p16. However, exceptions exist. Cytogenetic methods have also been explored and it has 


\section{Kiuru et al.}

been documented that a 4 probe FISH assay targeting RREB1, MYB, Cep6 and CGND1, ${ }^{16,17}$ can help in this setting albeit with limited sensitivity. While a positive test would support the diagnosis of melanoma, a negative result does not exclude melanoma. A melanoma may have copy number changes involving chromosomes not tested by this assay.

Apart from properties of the tumor cells (histopathologic appearance, immunophenotype, cytogenetic findings), the diagnostic evaluation of a sclerosing melanocytic proliferation also takes into account stromal findings (e.g. presence or absence of solar elastosis) and inflammatory changes. With regard to the latter, lymphocytic aggregates are commonly mentioned as a helpful diagnostic feature for the diagnosis of desmoplastic melanoma. . $^{1,2,8,9}$ However, little is known about the sensitivity and specificity of this feature for the distinction of desmoplastic melanoma from sclerosing melanocytic nevi. In a review of 20 randomly selected cases of desmoplastic melanomas, we found lymphocytic aggregates in 17 cases $(85 \%$; KJB; unpublished observations). Among 20 cases of randomly selected sclerosing nevi only three lesions (15\%) were associated with lymphocytic aggregates $(\mathrm{KJB}, \mathrm{RP}$; unpublished observations). While a more systematic assessment of this issue on a larger number of cases is desirable, it can be stated already that lymphocytic aggregates, albeit frequently present are not a constant feature of desmoplastic melanomas. Furthermore even if they are present, they may only be noted in a full thickness excision in the deeper portion of the tumor. It can also said that while lymphocytic aggregates seem to be present only in a minority of sclerosing nevi, they do occur, as documented herein. Thus, the feature per se is insufficient evidence for malignancy. The association of lymphocytic aggregates with a benign sclerosing melanocytic proliferations should not come as a surprise, because lymphocytic aggregates have been known to occur in association with an inflammatory fibrosing process, such as a scar or morphea. The findings in the current report do not diminish the value of lymphocytic aggregates prompting concerns about desmoplastic melanoma. However, our observation should caution pathologists to find additional evidence in support of or against melanoma before rendering a diagnosis. The presence of lymphocytic aggregates per se is simply not specific enough for melanoma.

\section{References}

1. Busam KJ. Cutaneous desmoplastic melanoma. Adv Anat Pathol 2005; 12: 92.

2. Busam KJ. Desmoplastic melanoma. Clin Lab Med 2011; 31: 321.

3. Busam KJ, Mujumdar U, Hummer AJ, et al. Cutaneous desmoplastic melanoma: reappraisal of morphologic heterogeneity and prognostic factors. Am J Surg Pathol 2004; 28: 1518.

4. Arora A, Lowe L, Su L, et al. Wide excision without radiation for desmoplastic melanoma. Cancer 2005; 104: 1462.

5. Hawkins WG, Busam KJ, Ben-Porat L, et al. Desmoplastic melanoma: a pathologically and clinically distinct form of cutaneous melanoma. Ann Surg Oncol 2005; 12: 207.

6. Pawlik TM, Ross MI, Prieto VG, et al. Assessment of the role of sentinel lymph node biopsy for primary cutaneous desmoplastic melanoma. Cancer 2006; 106: 900.

7. Murali R, Zannino D, Synnott M, McCarthy SW, Thompson JF, Scolyer RA. Clinical and pathological features of metastases of primary cutaneous desmoplastic melanoma. Histopathology 2011; 58: 886.
8. McCarthy SW, Scolyer RA, Palmer AA. Desmoplastic melanoma: a diagnostic trap for the unwary. Pathology 2004; 36: 445.

9. McCarthy SW, Scolyer RA. Pitfalls and important issues in the pathologic diagnosis of melanocytic tumors. Ochsner J 2010; 10: 66.

10. Troxel DB. Pitfalls in the diagnosis of malignant melanoma: findings of a risk management panel study. Am J Surg Pathol 2003; 27: 1278.

11. Harris GR, Shea CR, Horenstein MG, Reed JA, Burchette JL Jr, Prieto VG. Desmoplastic (sclerotic) nevus: an underrecognized entity that resembles dermatofibroma and desmoplastic melanoma. Am J Surg Pathol 1999; 23: 786.

12. Nojavan H, Cribier B, Mehregan DR. Desmoplastic Spitz nevus: a histopathological review and comparison with desmoplastic melanoma. Ann Dermatol Venereol 2009; 136: 689.

13. Sherrill AM, Crespo G, Prakash AV, Messina JL. Desmoplastic nevus: An entity distinct from spitz nevus and blue nevus. Am J Dermatopathol 2011; 33: 35.
14. Koc MK, Sudogan S, Kavala M, Kocaturk E, Büyükbabani N, Altintas S. Desmoplastic spitz naevus can be mistaken for desmoplastic malignant melanoma and dermatofibroma. Acta Derm Venereol 2011; 91: 74.

15. Ferrara G, Brasiello M, Annese P, et al. Desmoplastic nevus: clinicopathologic keynotes. Am J Dermatopathol 2009; 31: 718.

16. Gerami P, Jewell SS, Morrison LE, et al. Fluorescence in situ hybridization (FISH) as an ancillary diagnostic tool in the diagnosis of melanoma. Am J Surg Pathol 2009; 33: 1146.

17. Gerami P, Beilfuss B, Haghighat Z, Fang Y, Jhanwar S, Busam KJ. Fluorescence in situ hybridization as an ancillary method for the distinction of desmoplastic melanomas from sclerosing melanocytic nevi. J Cutan Pathol 2011; 38: 329.

18. Kucher C, Zhang PJ, Pasha T, et al. Expression of Melan-A and $\mathrm{Ki}-67$ in desmoplastic melanoma and desmoplastic nevi. Am J Dermatopathol 2004; 26: 452.

19. Hilliard NJ, Krahl D, Sellheyer K. p16 expression differentiates between desmoplastic Spitz nevus and desmoplastic melanoma. J Cutan Pathol 2009; 36: 753. 